\title{
A novel method for gear reducer's transient temperature field analysis based on thermo-fluid interaction
}

\author{
Liu Wangjia ${ }^{1, a}$, Fu Hongyong ${ }^{1, b}$ and Chen Yunxia ${ }^{2, c}$ \\ ${ }^{1}$ Technology and Engineering Center for Space Utilization, Chinese Academy of Sciences, Beijing \\ 100094, China; \\ ${ }^{2}$ School of Reliability and System Engineering, Beihang University, Beijing 100191, China. \\ alwj@csu.ac.cn, bfuhongyong@126.com, cchenyunxia@buaa.edu.cn
}

Keywords: Gear reducer, temperature field, thermo-fluid interaction, fluent

\begin{abstract}
In this paper, we focus on the need for temperature field analysis of gear transmission, as a important issue related to gear transmission's reliability. Most of previous studies were based on traditional heat transfer theory, focusing on numerical analysis method, however oil cooling effect has not been included in consideration. Based on CFD, this paper proposed a thermo-fluid coupled transient temperature field analysis method to a single reduction gear unit with oil bath lubrication and cooling conditions. This paper proceeds as follow. Firstly, the characteristics of gear reducer's heat generation and heat dissipation was analized, then utilize RNG $k-\varepsilon$ turbulence model to simulate the typically strong swirl inside the flow field, take advantage of implicit algorithm VOF model to accurately captures instantaneous changes of internal oil-gas interface. In the end, dynamic mesh was utilized to tracking the oil fluid during the process of gear movement. The heat gener-ated by gear set was calculated through analytic method and then impacted to gear surfaces in the simulation by user defined functions. Thus an effective model and method using Fluent software for direct simulation of gear reducer transient temperature field analysis is proposed, research is an important supporting role on the reliability and longevity of gear structure.
\end{abstract}

\section{Introduction}

Gear reducer is the most common form of mechanical transmission. Because of the friction between the gears, it is necessary to concern the gear system temperature field to ensure that the gear structure is not overheating, ensure the integrity of the structure of the gear to avoid performance degradation caused by overheated oil (viscosity reduction) and accidents (such as wind generators' lubricating oil has a chance of on fire). At the same time, the thermal stress caused by temperature rise is a necessary condition for thermal fatigue analysis and calculation of gear and gear shaft, even the bearings and housing. CFD simulation can be an effective approach for such investigation. For gear reducer simulation under the condition of oil bath lubrication, scholars have carried out research of different emphasis. Kris Riemslagh et al. [1] offered a method that allows the simulation of the flow of an incompressible fluid through rotary displacement pumps based on arbitrary Lagrangian-Eulerian formulation Navier-Stokes equations; Guillaume Houzeaux [2] presented a numerical strategy for the simulation of rotary positive displacement pumps which include dividing a cycle into a certain number of time steps and obtaining different computational meshes for each of these time steps; F.Lemfeld et al. [3] pioneered the use of two-phase flow model to capture the transient changes of fluid in the gear box, however he dealt with grid in a relatively simple way that gear tooth removed, while a certain wall roughness is a substitute for gear tooth, so oil churning can be observed; In 2011 V.Chernoray et al. [4] designed special rig for modelling a generic simplified gearbox with a single isolated rotating wheel, measured velocity distribution around the wheel and details on bubble and drop distribution. Su Hong [5] compared different simulation methods which aimed at finding the characteristics and practicability, using the immersed solid method and RFR provided by CFX, and dynamic grid method provided by Fluent to simulate the internal flow field of the gearbox. They explored the overall gear structure's oil lubrication, gained useful conclusions, but 
simulations for temperature rise problems caused by gear friction heat were not involved in many literatures yet. On this part of problem, H.Blok [6] derived formulas for calculating flash temperatures and instantaneous temperature rise for rubbing components that are prone to scuffing, such as highly loaded gear teeth in 1937. Townsend et al. [7] in 1981 performed a gear tooth temperature analysis using a finite element method combined with a calculated heat input, calculated oil jet impingement depth, and estimated heat transfer coefficients; in 1993 Anifantis et al. [8] put forward that film breakdown is due to overheating of gear teeth; in 2005 Atan and Ozdemir [9] established a multilayer feedforward neural network model to compute the asperity temperature rise for a given set of gears; Carlo Gorla et al. [10] in 2013 performed simulations in order to understand the influence of geometrical and operating parameters on the losses in power transmissions; Dong Chunfeng [11] has concentrated to obtaining contour of convective heat transfer coefficient of gears based on Fluent, and carried out temperature contour of gears, however in his study lubricating oil is neglected in some degree. Above work of simulation, although made some achievements that can be used to estimate the gear temperature field, but have not treat the gearbox oil and gear as a whole system. However, according to [12], 91\% of heat dissipation of the gearbox is through the oil and air convection, therefore, if an accurate understanding of that the gearbox cooling model is essentially a CFD model is established, the analysis accuracy and cost efficiency will be much superior to former methods.

This paper analyzed typical single-stage gear reducer's (including housing, gears, oil) transient temperature field with oil bath lubrication condition based on the finite volume method and ANSYS Fluent software, in which fluid is the simulation's principal part. In this paper, a simplify method for three-dimensional CAD model is studied, the reasonable constraints boundary conditions were studied, and turbulence, multiphase flow, moving mesh model to achieve gearbox thermal-fluid coupled flow simulation were investigated, thus a complete process of method of temperature field and flow field analysis for general oil bathed gear transmission structure was formed.

\section{Gear Reducer Numerical Model and the Fluid Governing Equations}

Typical gear reducer structure includes a housing, hubcaps, bearings, gears and shafts, shown in Figure 1:

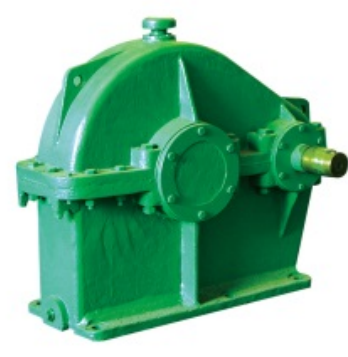

a real gearbox



b.CAD model

Fig 1. real single-stage gear reducer and its CAD model

It is usually powered by a driving shaft, the power pass through a single-stage gear, which include a pinion(driving gear) and driven gear, and transport to other machine by the driven shaft.

Finite Element Equations. Descriptions of flow and heat transfer in the form of partial differential equations have developed a number of ways to get the exact solution in mathematical community, but the exact analytical solution can only be obtained in some simple case. For practical engineering problems, numerical methods are widely used. First and foremost step is to establish governing equations.

Temperature Field Governing Equation. When creating a physical model of the gear reducer thermodynamics, we must consider the combination of the simulation and boundary conditions limits. When treat the friction heat of gears and bearings as an input source term, the gear reducer's transient temperature field governing equation are as follows [13]: 


$$
\frac{\partial}{\partial t}(\rho E)+\nabla \cdot(\vec{v}(\rho E+p))=\nabla \cdot\left(k_{e f f} \nabla T-\sum_{j} h_{j} \vec{J}_{j}+\left(\overline{\bar{\tau}}_{e f f} \cdot \vec{v}\right)\right)+S_{h}
$$

Where $k_{\text {eff }}$ is the effective conductivity( $k+k_{t}$, where $k_{t}$ is the turbulent thermal conductivity, defined according to the turbulence model being used), and $\vec{J}_{j}$ is the diffusion flux of species $j$. The first three terms on the right-hand side of equation represent energy transfer due to conduction, species diffusion, and viscous dissipation, respectively. $S_{h}$ is the heat of volumetric heat sources we defined through user defined fuctions(UDF).

Fluid Governing Equations. In general, conservation law for compressible Newtonian fluid has following descriptional equations [14]:

$$
\begin{aligned}
& \frac{\partial \rho_{f}}{\partial t}+\nabla \cdot\left(\rho_{f} \boldsymbol{v}\right)=0 \\
& \frac{\partial \rho_{f} \boldsymbol{v}}{\partial t}+\nabla \cdot\left(\rho_{f} \boldsymbol{v}-\tau_{f}\right)=f_{f}
\end{aligned}
$$

Where $t$ is time, $f_{f}$ is volume force vector, $\rho_{f}$ is density of fluid, $v$ is velocity vector of fluid, $\tau_{f}$ represents shear stress tensor, which can be expressed as:

$$
\tau_{f}=\left(-p-\frac{2}{3} \mu \nabla \cdot v\right) \delta+2 \mu e
$$

Where $\delta$ is strain tensor, $p$ is fluid pressure, $\mu$ is dynamic viscosity, $e$ is velocity stress tensor:

$$
e=\frac{1}{2}\left(\nabla \cdot \boldsymbol{v}+\nabla \cdot \boldsymbol{v}^{T}\right)
$$

Solid Governing Equation. The solid part of the conservation equation can be derived by Newton's second law:

$$
\rho_{s} d_{s}=\nabla \cdot \sigma_{s}+f_{s}
$$

Where $\rho_{s}$ is density of solid, $\sigma_{s}$ is Cauchy stress tensor, $f_{s}$ is Volume force vector, $d_{s}$ is solid domain local acceleration vector.

For the fluid part, total enthalpy form of the energy equation can be written in the following form:

$$
\frac{\partial\left(\rho h_{t o t}\right)}{\partial t}-\frac{\partial p}{\partial t}+\nabla \cdot\left(\rho_{f} v h_{t o t}\right)=\nabla \cdot(\lambda \nabla T)+\nabla \cdot(v \nabla \tau)+v \cdot \rho f_{f}+S_{E}
$$

Where $\lambda$ represents the thermal conductivity, $S_{E}$ is source term of energy.

$$
f_{T}=\alpha_{T} \cdot \nabla T
$$

Where $\alpha_{T}$ is the temperature-related thermal expansion coefficient.

Geometrical and Meshing Aspects. The complexity of three-dimensional structure increases the difficulty of meshing, as well as leads to unnecessary increase in the number of the grid, resulting the subsequent increased numerical computation. Since appropriate models are fundamental to simulations, the geometric processing tool SpaceClaim was utilized to simplify gear reducer's three-dimensional model: the shoulder of casing, hole, thrust washers are removed; wall thickness uniformed; rolling bearing structure taken by concentric rings at corresponding position, to facilitate applying heat source later [15]. As for gear pair, which are the core of gear reducer, no simplify applied to ensure the calculation's accuracy, except for enlarge centre distance to facilitate meshing. Ready model is shown in Fig.2: 


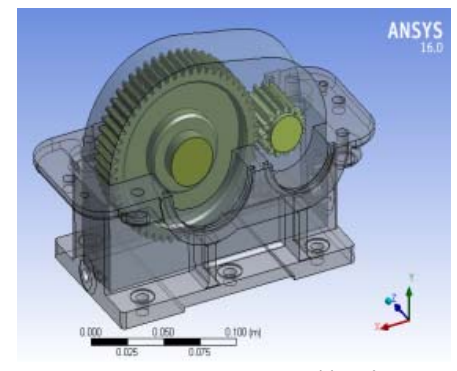

a.overall view

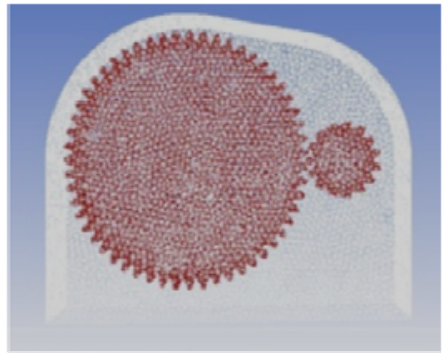

b.mesh view

Fig 2.overall view and mesh view of numerical model

Use ANSYS Meshing module for meshing, a certain refinement applied to the gear tooth mesh, the fluid and gear were attributed to different part, each mesh was meshed individually, which are connected at adjacent mesh with interface technology, and the details of the grid is shown in Figure 3:

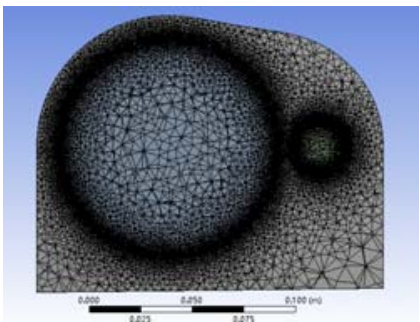

a.overall view



b.detailed view of mesh

Fig 3. overall view and detailed view of mesh

Heat Production Analysis. The heat rate on the tooth flank is calculated considering the friction losses. There are empirical models corresponding to this part of heat, and the correlation coefficient in the model can obtained in a relatively easy way. According to [16], the gear power losses can be subdivided into three parts, respectively sliding loss, rotationg loss, churning loss or windage loss.

$$
\begin{aligned}
& Q_{\text {gear }}=Q_{\text {slid }}+Q_{\text {roll }}+Q_{\text {wind }} \\
& Q_{\text {slid }}=0.0257 W_{N} v_{s} \log \left(\frac{29.66 W_{N}}{b \eta v_{s} v_{r}^{2}}\right) \\
& Q_{\text {roll }}=9 \times 10^{7} h v_{r} b \varepsilon_{\alpha} \\
& h=2.05 \times 10^{-7}\left(v_{r} \eta\right)^{0.67} W_{N}^{-0.067} \rho_{\text {eq }}^{0.464}
\end{aligned}
$$

Where $W_{N}$ is average normal load of meshing tooth face $(\mathrm{N}) ; v_{s}$ is average sliding velocityof meshing point $(\mathrm{m} / \mathrm{s}) ; v_{r}$ is average rotating velocity of meshing point $(\mathrm{m} / \mathrm{s}) ; b$ is tooth width $(\mathrm{m}) ; \eta$ is dynamic viscosity of oil $(\mathrm{Pa} \cdot \mathrm{s}) ; h$ is average oil film thickness of meshing tooth face $(\mathrm{m}) ; \varepsilon_{\alpha}$ is contact ratio of gear; $\rho_{e q}$ is equivalent radius of meshing point $(\mathrm{m})$.

Moreover, there are allocation relationship of rotating and sliding losses for gear pair:

$$
\left\{\begin{array}{l}
Q_{1}=\beta_{1} Q \\
Q_{2}=\left(1-\beta_{1}\right) Q
\end{array}\right.
$$

\section{Boundary and Simulation Model Analysis}

Research object is a complex combination of oil lubrication system, gear system and convective heat transfer systems. The heat of gear reducer comes form gear engagement zone and bearings, in general bearing heat accounts for about $1 \%$ of total heat production [15]. When the gear reducer is running at a certain working condition, the shaft and oil act as the heat transfer medium, transmit the heat to housing, which has an external air convection, as well as heat conduction with the mounting base. One can take advantage of the Fluent shell wall thickness treatment. By default, ANSYS Fluent treats walls as having zero thickness and presenting no thermal resistance to heat transfer across them. 
If a thickness is specified for a wall (thereby making it a thin wall, then the appropriate thermal resistance across the wall thickness is imposed.

Turbulence Model. Heat transfer process always depend on the mass transfer process(radiation not considered), therefore the flow degree of fluid in gear reducer simulation determines conjugate heat transfer degree. To consider this, it is required to investigate the form of the fluid motion on macroscopical. For selecting turbulent or laminar models, it is needed to determine the Reynolds number in the gear reducer's flow field. Reynolds number Re is calculated using the following formula:

$$
\operatorname{Re}=\frac{\rho v d}{\mu}
$$

Where $v, \rho, \mu$ is local velocity of fluid, density of fluid, kinetic viscosity respectively, $d$ is characteristic length. In the internal gear reducer, oil was thrown along the pitch circle, the maximum speed of oil is proximity of arbitrary point's line speed on gear pitch circle. Density of lubricating oil is according to the actual density; the viscosity coefficient, is the description of fluid viscosity, which can be obtained through the fluid attribute tables. Take the gear reducer's geometric characteristic length as characteristic length. When Reynolds number is relatively small, which means when fluid flow the viscous force between each particle is dominant, then fluid flow regularly, also known as laminar flow state; When Reynolds number is large, which means that the inertial forces is dominat, then fluid flow is turbulent state. In general $\operatorname{Re}<2300$ ascribe to laminar flow, $\operatorname{Re}=2300 \sim 4000$ ascribe to transition state, $\operatorname{Re}>4000$ is turbulent flow, $R e>10000$ is fully turbulent flow.

Staple turbulence flow models in Fluent include: Spalart-Allmaras model, $k-\varepsilon$ model, $k-\omega$ mode, where $k-\varepsilon$ model can be further divided into standard $k-\varepsilon$ model, Renormalization-group(RNG) model, $k-\varepsilon$ model with swirl modification. Two parameter RNG $k-\varepsilon$ model is chose, which is derived from the instantaneous Navier-Stokes equations, using a mathematical technique called "renormalization group" (RNG) methods [17]. The analytical derivation results in a model with constants different from those in the standard $k-\varepsilon$ model, and additional terms and functions in the transport equations for $k$ and $\varepsilon$. By calculating the Reynolds number, if they meet the turbulent conditions, the model can be used in the gear reducer flow field simulation.

The RNG $k-\varepsilon$ model has a similar form to the standard $k-\varepsilon$ model:

$$
\begin{aligned}
& \frac{\partial}{\partial t}(\rho k)+\frac{\partial}{\partial x_{i}}\left(\rho k u_{i}\right)=\frac{\partial}{\partial x_{j}}\left[\left(\mu+\frac{\mu_{t}}{\sigma_{k}}\right) \frac{\partial k}{\partial x_{j}}\right]+G_{k}+G_{b}-\rho \varepsilon-Y_{M}+S_{k} \\
& \frac{\partial}{\partial t}(\rho \varepsilon)+\frac{\partial}{\partial x_{i}}\left(\rho \varepsilon u_{i}\right)=\frac{\partial}{\partial x_{j}}\left[\left(\mu+\frac{\mu_{t}}{\sigma_{\varepsilon}}\right) \frac{\partial \varepsilon}{\partial x_{j}}\right]+C_{1 \varepsilon} \frac{\varepsilon}{k}\left(G_{k}+C_{3 \varepsilon} G_{b}\right)-C_{2 \varepsilon} \rho \frac{\varepsilon^{2}}{k}+S_{\varepsilon}
\end{aligned}
$$

In these equations, $G_{k}$ represents the generation of turbulence kinetic energy due to the mean velocity gradients, calculated as described in modeling turbulent production in the $k-\varepsilon$ models. $G_{b}$ is the generation of turbulence kinetic energy due to buoyancy, calculated as described in effects of buoyancy on turbulence in the $k-\varepsilon$ models. $Y_{M}$ represents the contribution of the fluctuating dilatation in compressible turbulence to the overall dissipation rate, calculated as described in effects of compressibility on turbulence in the models. The quantities $\sigma_{k}$ and $\sigma_{\varepsilon}$ are the inverse effective Prandtl numbers for $k$ and $\varepsilon$, respectively. $S_{k}$ and $S_{\varepsilon}$ are user-defined source terms.

Multi-phase Flow Model. Through the introducing of Multi-phase flow model, one can simulate inner environment of gear reducer in which oil and air exist simutaneously, then solve the coupled energy equations of both solid field and fluid field to caculate heat flow of gear reducer. Certain principles should be followed for selecting multiphase flow model according to the flow characteristics. For temperature field simulation of gear reducer, the multi-phase flow model which we employ should support for solving energy equations, as well as capture the dynamic liquid level accurately. Based on the above considerations, volume of fluid(VOF) model is chose in this study. 
For VOF model, Fluent solve volume fraction equation and energy equation, etc. The tracking of the interface between the air and oil is accomplished by the solution of a continuity equation for the volume fraction of one primary phase. For the $q^{\text {th }}$ phase, this equation has the following form:

$$
\frac{1}{\rho_{q}} \cdot\left[\frac{\partial}{\partial t}\left(\alpha_{q} \rho_{q}\right)+\nabla \cdot\left(\alpha_{q} \rho_{q} \vec{v}_{q}\right)\right]=\frac{1}{\rho_{q}} \cdot\left[S_{a_{q}}+\sum_{p=1}^{n}\left(\dot{m}_{p q}-\dot{m}_{q p}\right)\right]
$$

Where $m_{q p}$ is the mass transfer from phase $q$ to phase $p$ and $m_{p q}$ is the mass transfer form phase $p$ to phase $q$.

The energy equation, also shared among the phases, is shown below.

$$
\frac{\partial}{\partial t}(\rho E)+\nabla \cdot(\vec{v}(\rho E+p))=\nabla \cdot\left(k_{e f f} \nabla T\right)+S_{h}
$$

The VOF model treats energy, $E$, and temperature, $T$, as mass-averaged variables:

$$
E=\frac{\sum_{q=1}^{n} \alpha_{q} p_{q} E_{q}}{\sum_{q=1}^{n} \alpha_{q} p_{q}}
$$

Dynamic Mesh Model. Internal oil of the gear reducer plays double role of lubricating and cooling. To accurately simulate the oil movement surrounding the gear caused by spin of the gear, both dynamic grid zone and rigid zone is necessary at computing domain simutaneously. Mesh updating method contained in Fluent involve spring, Layering and Remeshing. Mesh updating is that solver updates the mesh due to the movement of the boundary of the computational domain during an iterative calculation process.

In this paper we employ spring and remeshing method to simulate the reconstruct of oil occupied grid cells. Mark and remesh the cells of which distortion is too large or change in the size is too drastic.

\section{Example of Transient Temperature Field Analysis of Gear Reducer}

Now with a single-stage gear reducer the transient temperature field in certain conditions were analyzed. The design parameters is outlined below, modulus is 2 , both pinion and driven gear is straight tooth gear, number of teeth $z_{1}, z_{2}$ is 15,55 respectively. The transmission ratio $u$ is 3.67 , centre distance $a_{d}$ is $70 \mathrm{~mm}$, the rotate speed of pinion varies from 1120 to $1680 \mathrm{rev} / \mathrm{min}$. In considering of that when two gears engage, no actual gap is between the tooth surface, the local dimension of flow field increases from zero, the minimum mesh size tends to be zero in the simulation. Therefore, enlarge the centre distance between two gears, the alteration is twice modulus namely $4 \mathrm{~mm}$. Tooth thickness of driven gear and pinion is $26 \mathrm{~mm}$ and $34 \mathrm{~mm}$ respectively.

Table 1 Turbulence model parameters

\begin{tabular}{lll}
\hline Model items & Parameters & Value \\
\hline Turbulence model & k-epsilon & $R N G$ \\
Near-wall treatment & Standard Wall Function \\
Model parameters & Cmu & 0.084 \\
& & 5 \\
& C1-Epsilon & 1.42 \\
& C2-Epsilon & 1.68 \\
& Swirl Factor & 0.07 \\
& Wall Prandtl Number & 0.85 \\
\hline
\end{tabular}


Table 2 Multi-phase model parameters

\begin{tabular}{llll}
\hline Model items & Parameters & Value \\
\hline Model & & Volume of Fluid \\
Volume Fraction Parameters & Volume Fraction Cutoff & le-06 \\
& Number of Eulerian Phases & 2 \\
& Body Force Formulation & Implicit Body Force \\
Materials & Phase & Air & Lubricant Oil \\
& Density $(\mathrm{kg} / \mathrm{m} 3)$ & 1.225 & 960 \\
& heat capability $(\mathrm{j} / \mathrm{kg} \cdot \mathrm{K})$ & 1006.43 & 1880 \\
& thermal conductivity $(\mathrm{W} / \mathrm{m} \cdot \mathrm{K})$ & 0.0242 & 0.12 \\
& Viscosity $(\mathrm{kg} / \mathrm{m} \bullet \mathrm{s})$ & $1.7984 \mathrm{e}-0$ & 0.048 \\
& & 5 & \\
& molecular weight $(\mathrm{kg} / \mathrm{kgmol})$ & 28.966 & 258.19 \\
& caloric content $(\mathrm{j} / \mathrm{kg} \cdot \mathrm{mol})$ & 0 & 0 \\
& Def.Temperature $(\mathrm{K})$ & 298.15 & 368.15 \\
\hline
\end{tabular}

Solving methods include selectiong of time step, iterative steps, the energy equation, momentum equation, spatial discretization, differential format. The complexity of the study and giant number of grid make it more difficult to converge, and a variety of models should also take into account that they can be easily converge, it should ensure equation convergence at the cost of some accuracy.

Table 3 Solving Methods

\begin{tabular}{lll}
\hline Model items & Parameters & Value \\
\hline Solve scheme & Pressure-Velocity Coupling & Simple \\
Spatial Discretization & Gradient & Least Squares Cell Based \\
& Pressure & PRESTO! \\
& Momentum & First Order Upwind \\
& Volume Fraction & Compressive \\
& Turbulent Kinetic Energy & First Order Upwind \\
& Turbulent Dissipation Rate & First Order Upwind \\
& Energy & Second Order Upwind \\
& Transient Formulation & First Order Implicit \\
\hline
\end{tabular}

Gear reducer's internal flow field on 0 s, $0.015 \mathrm{~s}, 0.03 \mathrm{~s}, 0.06 \mathrm{~s}$ is outlined in Fig 4:

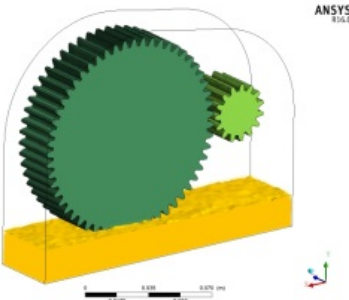

a. flow field on $0 \mathrm{~s}$

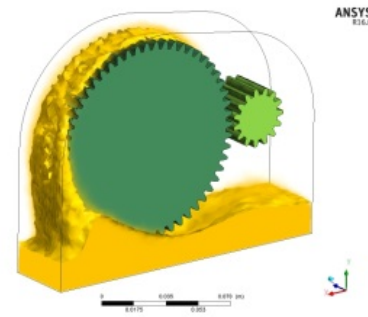

c. flow field on $0.03 \mathrm{~s}$



b. flow field on $0.015 \mathrm{~s}$



d. flow field on $0.06 \mathrm{~s}$

Fig 4. Internal flow field of gear reducer on different time

Contour of temperature field on 0.18 s, 0.36 s of pinion gear is outlined in Fig 5: 


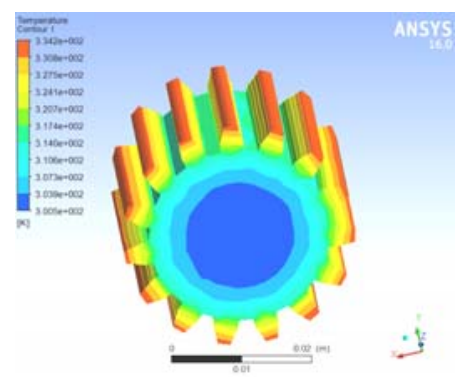

a. Contour on $0.18 \mathrm{~s}$



b. Contour on $0.36 \mathrm{~s}$

Fig 5. Contour of temperature field of pinion gear

Temperature-time history of the certain sampled point on driving gear is draw in two typical working conditions respectively in Fig 6:

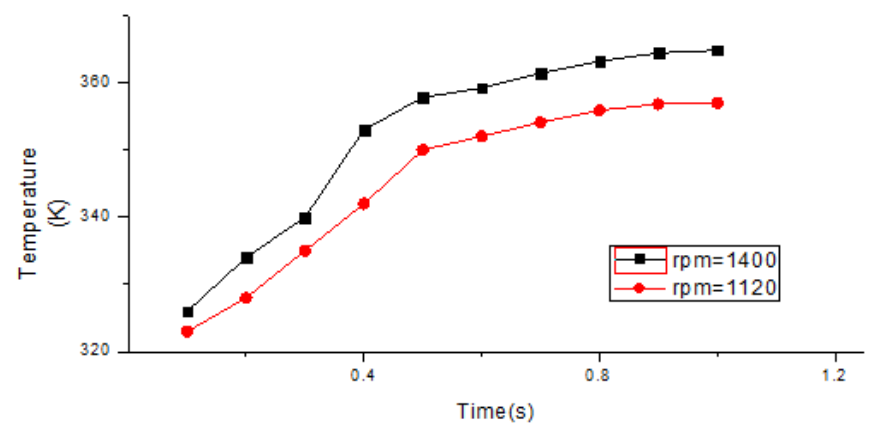

Fig 6. Temperature-time curve of sample point on pinion gear

Fig $5 \& 6$ show that the peak temperature under different working conditions on gear have a difference, and increases with time. Under high rotation speed, the temperature is higher than low rotation speed because heat flux generating rate of former is much larger.

Streamlines in the gearbox is shown in Fig 7:

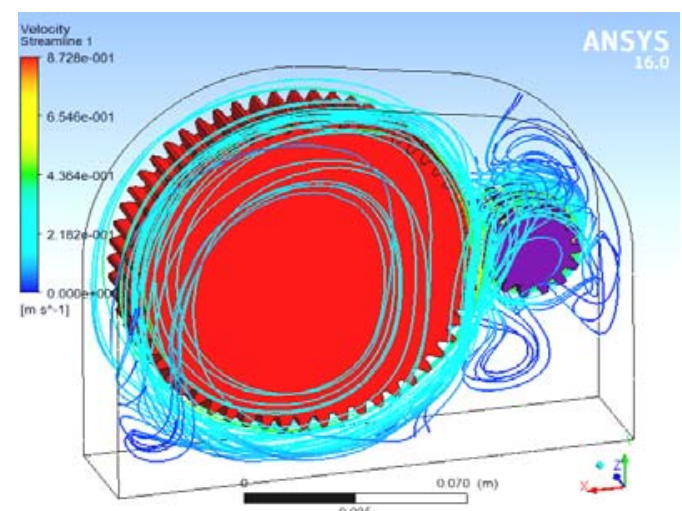

Fig 7. Streamlines in the gearbox on $0.3 \mathrm{~s}$

From Fig 7, it can be concluded that the flow field is swirl dominated, causing the inaccuracy of normal $k-\varepsilon$ turbulence model, therefore the introduc-ing of RNG $k-\varepsilon$ model is necessary.

\section{Summary}

1)Aiming at former gear transmission studies' problem that having not combined oil cooling with gear reducer itself as a organic whole, issue solved effectively through taking flow field as main body of simulation; results is beneficial both for design purposes(like predict power losses)and for the prediction of durable life.

2)Transient temperature field simulation method of gear reducer based on thermal-fluid coupling was proposed, at the same time transient temperature field simulation model of gear reducer using 
Fluent was established. By analyzing the basic rules and characteristics of gear reducer's heat production and dissipation, $\mathrm{RNG} k-\varepsilon$ turbulence model is adopted to simulate turbulence in gear reducer, VOF multi-flow model is adopted to simulate coexistence of oil and air, dynamic mesh and tetrahedron mesh was adopted to simulate oil splashing, real-time updating of oil and gas and gear solid's conjugate heat transfer area was realized under the premise of achieving the accurate simulation of the flow field. The analysis framework is applicable to the whole family.

3 ) Results of this paper has significance of reliability improving and lifetime evaluation to the gear reducer. Limited in hardware resources, this article currently only simulated single-stage gear reducer. If conditions permited, the multi-stage gear reducer and more number of grid can be simulated.

\section{References}

[1]Kris Riemslagh, Jan Vierendeels, Erik Dick 2000. An arbitrary Lagrangian-Eulerian finite-volume method for the simulation of rotary displacement pump flow, Applied Numerical Mathematics 32 (2000) 419-433.

[2]Guillaume Houzeaux, Ramon Codina 2007. A finite element method for the solution of rotary pumps. Computers \& Fluids 36 (2007) 667-679.

[3]Frantisek LEMFELD, Karel FRAŇA, Jiř́ UNGER 2007. Numerical simulations of unsteady oil flows in the gear-boxes, Journal of applied science in the thermodynamics and fluid mechanics Vol. 1, No. $1 / 2007$

[4]Chernoray, V., Jahanmiri. M 2011. Experimental study of multiphase flow in a model gearbox, WIT Transactions on Engineering Sciences, v 70, p 153-164, 2011, Computational Methods in Multiphase Flow VI.

[5]Su Hong 2014. Oil splashing simulation of single stage gear based on CFD [In Chinese]. Degree paper. Chongqing University.

[6]Blok H 1937. Theoretical study on temperature rise at surface of actual contact under oiliness lubrication conditions, Proc. Gen. Discussion on Lubrication , 2: 222-235.

[7]Townsend D P, Akin L S 1981. Analytical and experimental spur gear tooth temperature as affected by operating variables, Journal of Mechanical Design, Transactions of the ASME, 103(1): 219-226.

[8]Anifantis N, Dimarogonas A. D 1993. Flash and bulk temperatures of gear teeth due to friction, Mechanism and Machine Theory, Volume 28, Issue 1, January 1993, 159-164.

[9]Ebubekir Atan, Serhan Ozdemir 2005. Intelligence modeling of the transient asperity temperatures in meshing spur gears, Mechanism and Machine Theory 40 (2005) 119-127.

[10]CarloGorla, FrancoConcli, KarstenStahl, Bernd-RobertHöhn, KlausMichaelis, Hansjörg Schultheiß, Johann-Paul Stemplinger 2013. Hydraulic losses of a gearbox: CFD analysis and experiments, Tribology International 66(2013)337-344.

[11]Dong Chunfeng 2011. Heat-fluid coupled analysis on convection heat transfer and temperature field of gears [In Chinese]. Degree paper. Chongqing University.

[12]Fu Sinan 1988. Power loss and heat dissipation calculations of gear reducers[In Chinese], Machinery 1988,vol5.

[13]ANSYS Inc. 2014. ANSYS Fluent theory guide. Chapter 5 Heat transfer 168-170.

[14]John D. Anderson 2000.Computational Fluid Dynamics. Mc Graw Hill Education.

[15]Liu Wangjia 2014. Analysis of accessory gearbox's mechanical behavior and fatigue life evaluation considering thermal-structure coupling[In Chinese], Degree paper. Beihang University. 
[16]Anderson. Neil E, Loewenthal. Stuart H, Black. Joseph D 1984. Analytical method to predict efficiency of aircraft gearboxes, NASA Technical Memorandum. 1984.

[17]Yakhot V,Orszag SA,Thangam S,Gatski 1991. Development of turbulence models for shear flows by a double expansion technique, Physics of Fluids A 1991;4(7):1510-20. 